\title{
Las comunidades de habla en Barranquilla: nativos e inmigrantes del Caribe
}

\author{
Yolanda Rodríguez Cadena ${ }^{1}$ \\ Universidad del Atlántico
}

\section{Resumen}

En este ensayo se describen algunos fenómenos fónicos de la comunidad de habla barranquillera, tanto de los nativos como de los inmigrantes de las otras zonas del Caribe colombiano. También se establecen relaciones con el resto del Caribe, lo que demuestra la pertenencia de Barranquilla a esta gran zona dialectal. Las variables analizadas son la (s) y la (r), las cuales se relacionan con factores internos y externos. Dentro de los primeros están: el contexto en la palabra, el entorno fonológico, la tonicidad de la sílaba y el valor morfológico. Dentro de los segundos están: los factores sociales como el sexo, la edad, el nivel de instrucción y la procedencia; este último permite estudiar el comportamiento lingüístico de los inmigrantes y se analiza con la variable, tiempo de residencia. Los resultados demuestran que la variación fónica en Barranquilla obedece a dichos factores inter-

\section{Abstract}

In this essay some phonic features of the Barranquillera speaking community are described, the natives as well as the immigrants from other areas of the Colombian Caribbean. Relationships with the rest of the Caribbean are also established, which demonstrates Barranquilla belonging to this great dialectal area. The analyzed variables are the (s) and the (r), which are related with internal and external factors. The first ones such as: the context in the word, the phonological environment, the tonicity of the syllable and the morphological value. The second ones such as: the social factors as the sex, the age, the instruction level and the origin; this last one allows us to study the immigrants' linguistic behavior and it is analyzed with the variable, time of residence. The results demonstrate that the phonic variation in Barranquilla is influenced by these internal and

\footnotetext{
1 Magíster en Lingüística Española del Instituto Caro y Cuervo. Magíster en Lingüística y candidata a Doctor por el Colegio de México. Actualmente es docente de la Universidad del Atlántico. Correo-e: yolarca@hotmail.com.
} 
nos y externos; y que se están presentando procesos de desdialectalización en los inmigrantes.

Palabras clave: factores lingüísticos y sociales, dialecto, variación, comunidad de habla. external factors; and that de-dialectalization processes are being presented in the immigrants.

Keywords: linguistic and social factors, dialect, variation, speech community.

La consolidación de las migraciones en Barranquilla ocurre en los comienzos del siglo XX, cuando la ciudad obtuvo un desarrollo social, económico, demográfico y cultural considerable y vertiginoso comparado con el de otras ciudades de tradición histórica en el Caribe colombiano, como Cartagena y Santa Marta. La transformación de Barranquilla, de poblado de artesanos y comerciantes a cabeza de región y fuente de desarrollo y progreso durante la República, y luego a ciudad principal del Caribe colombiano, en menos de 50 años, la identifica históricamente entre todas las ciudades de Colombia. Desde la primera mitad del siglo XIX era sólo un puerto de exportación que luego se convirtió en lugar de importaciones². Esta condición de puerto y la actividad mercantil causó una ola de migración de grupos provenientes del resto del Caribe colombiano y de otras partes de Colombia: Bolívar, Magdalena, Norte de Santander, entre los principales, y grupos extranjeros de origen judío ${ }^{3}$, alemán, italiano, sirio, libanés y palestino ${ }^{4}$.

\footnotetext{
${ }^{2}$ El crecimiento demográfico se debió, según Arteta y Atencio, al auge del comercio exterior del país, pues en Barranquilla se establecieron las principales casas de comercio y funcionaban numerosos bancos nacionales y extranjeros (1990: 5). Negociaban con ganado: L.A. Roncallo, Pacini y Puccini, Santo Domingo y Cía., D. J. Senior, Fuenmayor y Cortissoz; con cueros: Lascano y Cía, Antonio Volpe y Cía., Angulo y Cía.; en algodón: Mayans, Hermanos y Cía., La Compañía Agrícola y Comercial. Entre los bancos está el Banco Dugand (Posada Carbó, 1987: 89). Además de las compañías y bancos, vale la pena destacar que en Barranquilla dieciséis países tenían representación consular en 1892 (Posada Carbó, 1998: 329). La ciudad se convirtió así en el primer puerto del país, gracias además, a la construcción de un ferrocarril que la unía con Sabanilla (Atlántico) y a la construcción del muelle de Puerto Colombia.

${ }^{3}$ Se registra la entrada de familias judías a Barranquilla desde finales del siglo XIX y comienzos del XX; entre otras: las familias Cybulkiewics (1929-1930), Gontovnik (1930-1933), Kovalski (1928), Safdeye (1920) (Manco Bermúdez \& Watnik Barón, 1996: 65-173).

${ }^{4}$ En el censo de población de 1951 se ofrece la siguiente información sobre los inmigrantes de Barranquilla discriminados por departamentos de origen: Bolívar (45.766); Magdalena (30.419); Norte de Santander (6.095); Cundinamarca (4.938); Antioquia (4.481); Santander (4.150), Tolima (2.526); Caldas (1.281); Boyacá (1.109); Valle (999); Chocó (268); Nariño (252); Cauca (172). Lo interesante aquí es
} 


\begin{tabular}{|c|c|}
\hline AÑO & POBLACIÓN \\
\hline 1843 & 5.651 \\
\hline 1851 & 6.114 \\
\hline 1870 & 11.595 \\
\hline 1918 & 64.543 \\
\hline 1938 & 152.348 \\
\hline 1973 & 665.917 \\
\hline 1993 & $1 ' 348.536$ \\
\hline
\end{tabular}

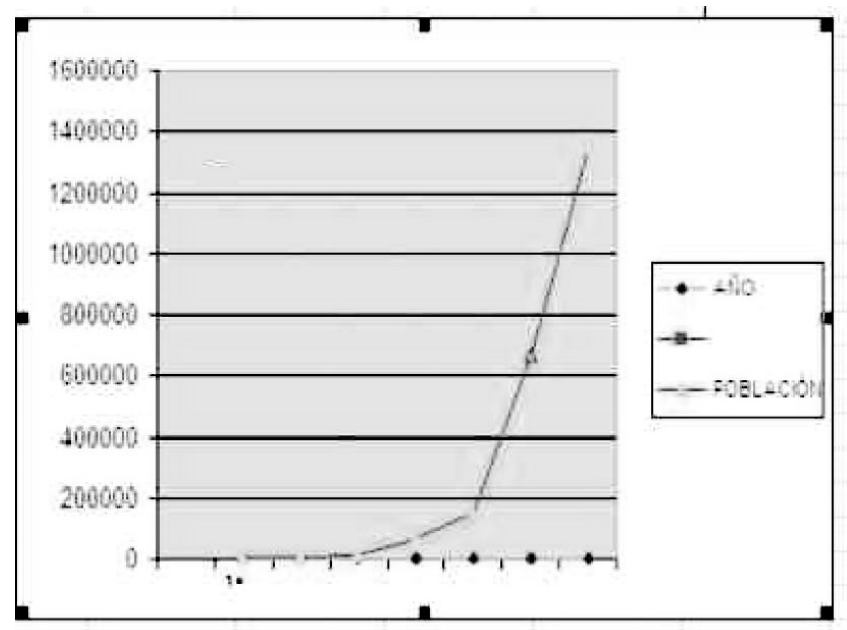

Gráfica 1. Crecimiento de la población en Barranquilla.

Siglos XIX y XX. En miles de habitantes.

El perfil sociocultural y lingüístico de Barranquilla se consolidó gracias a la intensa actividad económica, tal como afirman Arteta y Atencio (1990: 7): "En este sentido, se puede decir que Barranquilla es una 'muestra' de la Costa, pues en ella confluyen habitantes de todos sus rincones. De ahí que se haya constituido en un mosaico de costumbres, tradiciones y valores". La diversidad cultural y de grupos de diferentes orígenes geográficos generó una diversidad de comunidades dialectales, sociales y bilingües ${ }^{5}$ (ver esquema 1 ).

que para ese año el total de inmigrantes era de 103.184, y de nacidos en Barranquilla, de 279.627, un 26.9 \% de la población (Havens \& Usandizaga, 1966: 20, citado por Posada Carbó, 1987: 87). Hoy en día dicho porcentaje asciende al 30 \%, según el censo de 1993. Con respecto a los extranjeros, es necesario mencionar que muchos de los fundadores de las casas comerciales eran inmigrantes judíos, como los Senior, alemanes como los Gieseken, italianos como los Pacini. En 1894 se fundó la firma Bichar Jassir Hermanos, que marcó el inicio de otra ola de inmigrantes, de sirios, libaneses y palestinos (Posada Carbó, 1987: 89-90).

${ }^{5}$ Con respecto a la presencia de lenguas diferentes al español en Barranquilla, vale la pena mencionar el comentario que hace Posada Carbó (1998: 329) sobre los alma- 


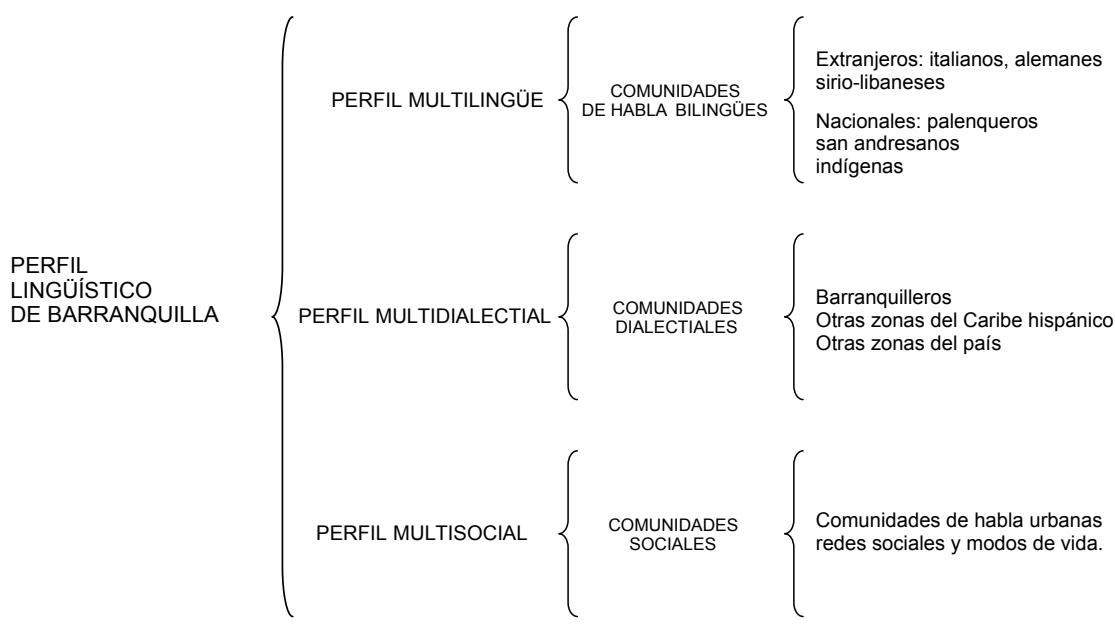

Esquema 1. Perfil lingüístico de Barranquilla.

Conviven, pues, en la ciudad grupos extranjeros cuya lengua materna se ha relegado a las interacciones íntimas y familiares, o en otros casos, se ha perdido a favor de un monolingüismo en español, en especial en las últimas generaciones. Y en cuanto a las comunidades hispanas, en Barranquilla también residen grupos del Caribe colombiano y del interior del país. De estas comunidades es importante señalar al grupo sanandresano y al grupo de palenqueros ${ }^{6}$. El primero está conformado por personas que inmigraron a Barraquilla por motivos de estudio. Muchos residen un tiempo considerable y luego regresan a San Andrés; otros, en cambio, toman la ciudad como lugar de residencia estable, cuando se han ubicado laboralmente y han conformado un núcleo familiar. La comunidad palenquera, por su parte, está constituida por familias que emigraron a Barranquilla en las décadas de los treinta, cuarenta y cincuenta del siglo XX, motivados por el auge económico de la ciudad. Las comunidades provenientes de las otras zonas del Caribe emigraron aproximadamente en las mismas épocas en que lo hicieron los palenqueros, y aún siguen desplazándose a la ciudad siempre en busca de mejores condiciones económicas y educativas.

Todas estas comunidades comparten hoy en día espacios, situaciones e interacciones sociales y lingüísticas, y están integradas a una

cenes: "Los almacenes de la Plaza de San Nicolás, como Baena Hermanos, anunciaban en sus puertas: «English spoken, on parle francais, si parla italiano»". También menciona Posada Carbó (1998: 329) que el Boletín Municipal de Estadística algunas veces publicaba artículos y anuncios en idiomas extranjeros; y en 1872, Elías Pellet fundó el periódico comercial editado en inglés The Shipping List.

${ }^{6}$ Los palenqueros son originarios de San Basilio de Palenque, un asentamiento negro situado a 60 kilómetros de Cartagena. 
estructura socioeconómica, unos modos de vida y redes sociales específicas. Comparten, además, normas dialectales del Caribe como la aspiración y elisión de la [s] y [r], la velarización de la [n] en posición final de palabra y otra serie de fenómenos pertenecientes a los mismos procesos de debilitamiento consonántico. Con base en estos hechos se puede aseverar, siguiendo a Gumperz (1971: 116), que, pese a las diferencias lingüísticas -que para el caso de Barranquilla provienen de los procesos migratorios causados por el desarrollo económico-, las variedades lingüísticas usadas dentro de la comunidad de habla forman un sistema, pues se vinculan a conjuntos de normas sociales, normas de producción e interpretación lingüísticas puestas en escena en el contacto diario.

De estos inmigrantes vale la pena destacar a la comunidad palenquera, la cual está conformada por un grupo étnica, territorial y socialmente diferenciado, que demuestra un sentido de pertenencia a la ciudad de Barranquilla, comparte la variedad dialectal del Caribe hispánico y forma parte de la identidad caribeña ${ }^{7}$. Esta pertenencia se constata en las entrevistas realizadas, especialmente en las pruebas de evaluación subjetiva, en las cuales el sentido de identidad y lealtad con respecto a la ciudad sobrepasa al de etnicidad. En el caso de los grupos provenientes del resto del Caribe, el sentido de pertenencia e identidad con la comunidad de habla barranquillera es mayor y más marcado, pues la barrera étnica no existe y se asimilan rápidamente al contexto urbano, llegando a perder incluso sus marcas dialectales específicas de la subregión caribeña de origen; su variedad de lengua deja de ser enfocada para pasar a ser difusa ${ }^{8}$. Esto ocurre con mayor frecuencia cuando el subdialecto caribeño del inmigrante no nacido en Barranquilla se aleja considerablemente del de la ciudad y cuando posee rasgos lingüísticos estigmatizados ${ }^{9}$. Estos procesos

\footnotetext{
${ }^{7}$ Esta identidad se manifiesta en la participación étnica del grupo negro en el mestizaje, en las prácticas culturales que forman parte del Caribe como la música y la danza (cumbia, mapalé), fiestas de santos, velorios, entre otros (De Friedemann, 1992: 547). Este grupo étnico también ha participado en la formación del español caribeño.

${ }^{8}$ Una variedad de lengua es enfocada cuando sus hablantes la perciben como una entidad en cierto sentido distinta, mientras que es difusa en los casos en que los patrones de lengua de personas social y geográficamente móviles no se pueden caracterizar como pertenecientes a algún acento particular, sino que son una mezcla de varios acentos regionales y sociales. Con respecto al concepto "acento", Milroy considera que es una noción más psicosocial que estrictamente lingüística; los hablantes muestran una conciencia de su importancia como marcadores de varios aspectos de la identidad social (Milroy, 1982: 141).

${ }^{9}$ La variedad del español del Caribe hablado en Barranquilla es considerada prestigiosa en la región y no posee rasgos fónicos estigmatizados por los hablantes como
} 
de desplazamiento dialectal o desdialectalización se verificarán más adelante con las variables lingüísticas seleccionadas en el análisis.

En este ensayo se estudian los inmigrantes del Caribe colombiano llegados a Barranquilla en diferentes épocas y con residencia relativamente estable. La migración que analicé como proceso sociolingüístico revela dos hechos claves que se corroborarán en el análisis: el primero es la incidencia de la comunidad de habla barranquillera con sus características lingüísticas sobre las comunidades inmigrantes, lo cual marca los procesos de variación y cambio lingüístico causantes de la desdialectalización (desplazamiento de dialecto) en los hablantes debido a varios factores. Este desplazamiento tiene su contraparte -al igual que en las situaciones de bilingüismo- en el mantenimiento del dialecto debido también a diversas causas psicosociales. El segundo hecho es la diversidad lingüística que se constituye en un rasgo de la ciudad de Barranquilla en la que se pueden encontrar muestras de los diferentes dialectos del Caribe colombiano interactuando, traslapándose, en juego polifónico de culturas y subculturas. Estos hechos se analizarán a continuación.

Para cumplir con el objetivo de este ensayo he seleccionado una parte del resultado de la investigación completa titulada Las comunidades de habla en Barranquilla: nativos e inmigrantes, estudio sociolingüístico que se desarrolló durante el año 2004 en el marco de la convocatoria Pensar el Caribe de la Universidad del Atlántico. Esta parte del resultado describe y explica desde la sociolingüística variacionista algunos procesos de variación y cambio lingüístico en la ciudad de Barranquilla. Se analizan, especialmente, la variable (s) implosiva con sus variantes: plena [s], aspirada [h], elidida [0] y asimilada total al segmento siguiente [a]; y la variable (r) implosiva, con sus variantes: plena [r], asimilada total al segmento siguiente [a] y elidida [0]. Las variables sociales independientes tomadas son: sexo, edad, nivel educativo, etnia, origen y tiempo de residencia en la ciudad. En el factor edad se tuvieron en cuenta cuatro grupos generacionales: grupo 1 , de 16 a 20 años; grupo 2, de 30 a 50 años; grupo 3, de 51 a 60 años; y grupo 4, de 61 años en adelante. Los niveles educativos analizados son: nivel 1 , con educación primaria; nivel 2, con educación secundaria, y nivel 3, con educación universitaria. Finalmente, la variable etnia se tomó en

la asimilación total al segmento siguiente de las líquidas en posición implosiva, el cual caracteriza los subdialectos de los departamentos caribeños de Córdoba, Sucre y Bolívar. Los mismos hablantes barranquilleros consideran que su variedad del español es prestigiosa frente a las otras del Caribe; consideran que no hablan "golpeado" (término para referirse a la asimilación total al segmento siguiente de líquidas) ni "cantado" ni "gritado" (término para referirse a la entonación especial y marcada de los habitantes de La Guajira y El Cesar, departamentos del Caribe). 
el sentido de etnicidad, tal y como la plantea Labov (2001: 245), como un rasgo que representa una identidad social atribuida a la gente en virtud de sus descendientes, como una característica adquirida que responde a la pregunta “¿qué eres?”. Muchas veces estos grupos se cohesionan alrededor de filiaciones religiosas, estructuras de creencias e incluso áreas territoriales. Estos factores sociales independientes se relacionarán con las variables fónicas en sus variantes, en los planos cualitativo y cuantitativo.

De los 127 informantes entrevistados en el proyecto general se seleccionaron 32 entrevistas de 32 informantes con una duración de una hora, del tipo semidirigido, basado en un módulo de temas que permitieron generar la espontaneidad en la conversación con los informantes; pero, además del carácter informal, se deslindaron los momentos de semiformalidad de la conversación marcados discursivamente. Veamos los resultados.

\section{Variable (s)}

El español de la ciudad de Barranquilla registra cuatro variantes de (s), como se anotó antes: plena [s], aspirada [h], elidida [0] y asimilada plena al segmento siguiente [a]. Para el análisis lingüístico tomé tres contextos: la posición interior y final; el contexto fonológico final ante vocal $(-\mathrm{V})$, ante consonante $(-\mathrm{C})$ y ante pausa $(-/)$; la tonicidad de la sílaba, tónica y átona, y el valor morfológico de (s). Los resultados cuantitativos revelan las variantes que identifican la comunidad de habla barranquillera y las restricciones de aparición de las variantes. Se tomaron 100 casos de (s) de cada informante para un total de 3.200 casos.

\begin{tabular}{|c|c|c|c|c|c|}
\hline \multicolumn{3}{|c|}{ Barranquilla } & \multicolumn{3}{c|}{ Puerto Rico } \\
\hline & $\begin{array}{c}\text { Número de } \\
\text { casos }\end{array}$ & $\%$ & & $\begin{array}{c}\text { Número de } \\
\text { casos }\end{array}$ & $\%$ \\
\hline $\mathrm{s}$ & 504 & 15.7 & $\mathrm{~s}$ & 2.235 & 9.0 \\
\hline $\mathrm{h}$ & 1.370 & 42.8 & $\mathrm{~h}$ & 12.705 & 51.1 \\
\hline 0 & 1.319 & 41.2 & 0 & 9.489 & 38.2 \\
\hline $\mathrm{a}$ & 7 & 0.2 & $\mathrm{a}$ & 398 & 1.5 \\
\hline
\end{tabular}

Tabla I. Variantes de (s) en Barranquilla y Puerto Rico.

Las variantes de (s) más frecuentes en Barranquilla son la aspirada y la elidida; los porcentajes reflejan la pugna entre la dos normas de uso; la retención de (s) plena es menor; y la asimilación plena revela un porcentaje mínimo. Estos resultados son análogos a los hallados en Puerto Rico (López Morales, 1983: 39) aunque la norma de 
elisión es más frecuente en Barranquilla. La posición revela también hechos interesantes en Barranquilla que se pueden comparar con otras zonas del Caribe.

\begin{tabular}{|c|c|c|}
\hline Variante Posición $\mathbf{t}+$ & Interior $\%$ & Final $\%$ \\
\hline $\mathrm{s}$ & 12.5 & 16.9 \\
\hline $\mathrm{h}$ & 69.1 & 32.7 \\
\hline 0 & 17.4 & 50.3 \\
\hline $\mathrm{a}$ & 0.7 & 00.0 \\
\hline Número de casos & 886 & 2.314 \\
\hline
\end{tabular}

Tabla 2. Distribución de las variantes de (s) según la posición.

La posición refleja datos importantes para las variantes aspirada y elidida; la primera está favorecida por la posición interior (69.1\%), mientras que la segunda por la posición final (50.3\%). La posición interior es desfavorable para la elisión, y la final para la aspiración; se aprecia una distribución complementaria de las variantes. En cuanto a la [s] plena, las frecuencias no establecen diferencia significativa, aunque es la posición final la que muestra mayor frecuencia. La asimilada plena al segmento siguiente fue poco frecuente en el corpus tomado, lo que indica que no es una variante que caracterice la comunidad de habla barranquillera, como si lo son la aspiración y la elisión.

\begin{tabular}{|c|c|c|c|c|c|c|}
\hline & \multicolumn{2}{|c|}{ SAN JUAN } & \multicolumn{2}{c|}{ PANAMÁ } & \multicolumn{2}{c|}{ SANTIAGO } \\
\hline & Interior $\%$ & Final $\%$ & Interior \% & Final \% & Interior \% & Final \% \\
\hline $\mathrm{s}$ & 7.4 & 9.6 & 2 & 14 & 10 & 11 \\
\hline $\mathrm{h}$ & 80.4 & 43.8 & 57 & 36 & 29 & 20 \\
\hline 0 & 11.6 & 46.5 & 41 & 50 & 61 & 69 \\
\hline
\end{tabular}

Tabla 3. Distribución de las variantes de (s) en varios dialectos del Caribe.

Mis resultados son análogos a los de San Juan de Puerto Rico (López Morales, 1983) y Panamá (Cedergren, 1973). La posición interior de palabra es la más favorable para la aspiración, y la posición final, para la elisión. En el español dominicano de Santiago (Alba, 1990) se registra el mismo patrón. Pero las diferencias entre la elisión y la aspiración en las posiciones interior y final son menos marcadas.

El contexto fonológico también presenta resultados interesantes en Barranquilla. Veamos: 


\begin{tabular}{|c|c|c|c|}
\hline & $-\mathrm{C} \%$ & $-\mathrm{V} \%$ & $-/ \%$ \\
\hline $\mathrm{s}$ & 9.8 & 18 & 44.8 \\
\hline $\mathrm{h}$ & 35.8 & 42.4 & 1.2 \\
\hline 0 & 54.2 & 39.5 & 53.9 \\
\hline
\end{tabular}

Tabla 4. Distribución de las variantes de (s) según el contexto fonológico.

El contexto fonológico que más favorece la aspiración es el prevocálico; la elisión se favorece en los contextos preconsonántico y prepausal; el contexto que menos favorece la aspiración es el prepausal; nuevamente se encuentra una distribución complementaria en la [h] y la [0], en cuanto al entorno fonológico. La retención de [s] plena, pese a su poca frecuencia en la comunidad de habla barranquillera, se favorece en el contexto prepausal. Es interesante ver que la elisión presenta aún un índice alto en el contexto prevocálico (39.5) cercano al de la aspiración. Esto revela la fortaleza de esta regla de elisión en esta comunidad, la cual es susceptible de aparecer en cualquier contexto fónico. Veamos algunos datos de otras zonas del Caribe:

\begin{tabular}{|c|c|c|c|}
\hline & $-\mathrm{C} \%$ & $-\mathrm{V} \%$ & $-/ \%$ \\
\hline La Habana & 21 & 18 & 61 \\
\hline Caracas & 10 & 50 & 40 \\
\hline Merida & 21 & 20 & 59 \\
\hline San Juan & 5.8 & 17.9 & 10.5 \\
\hline Panamá & 5 & 20 & 34 \\
\hline Santiago & 3 & 21 & 18 \\
\hline
\end{tabular}

Nota: Datos tomados de López Morales (1992: 82-83) y Cedergren (1973: 43).

Tabla 5. Distribución de (s) plena en varios dialectos del Caribe.

Al comparar mis resultados con los de las otras comunidades del Caribe, se encuentran semejanzas: en La Habana, Mérida y Panamá el contexto fónico que más favorece el mantenimiento de [s] es el prepausal, al igual que en Barranquilla. El segundo contexto favorecedor es el prevocálico en Caracas, San Juan y Panamá, al igual que en Barranquilla; en Santiago es el entorno más favorecedor, y en segundo lugar lo es el prepausal.

\begin{tabular}{|c|c|c|c|}
\hline & $-\mathrm{C} \%$ & $-\mathrm{V} \%$ & $-/ \%$ \\
\hline Cuba & 75 & 48 & 13 \\
\hline San Juan & 55.1 & 41.3 & 20.1 \\
\hline Panamá & 45 & 30 & 16 \\
\hline Santiago & 23 & 21 & 15 \\
\hline
\end{tabular}

Nota: Datos tomados de López Morales (1992: 82-83); Cedergren (1973: 43); Terrell (1979: 602), y Alba (1990: 79).

Tabla 6. Distribución de (s) aspirada [h] en varios dialectos del Caribe. 
En las cuatro comunidades del Caribe citadas en la tabla 6, el contexto favorecedor de la aspiración es el preconsonántico, y en segundo lugar está el entorno prevocálico; en Barranquilla es el prevocálico el más favorecedor, aunque el preconsonántico es el segundo contexto favorecedor. Tanto en Barranquilla como en las comunidades del Caribe citadas en la tabla 6, el entorno menos favorecedor para la aspiración es el prepausal.

\begin{tabular}{|c|c|c|c|}
\hline & $-\mathrm{C} \%$ & $-\mathrm{V} \%$ & $-/ \%$ \\
\hline Cuba & 23 & 34 & 26 \\
\hline San Juan & 39 & 40.7 & 69.2 \\
\hline Panamá & 50 & 49 & 50 \\
\hline Santiago & 74 & 58 & 67 \\
\hline
\end{tabular}

Nota: Datos tomados de López Morales (1992: 82-83); Cedergren (1973: 43); Terrell (1979: 602), y Alba (1990: 79).

Tabla 7. Distribución de (s) elidida [0] en varios dialectos del Caribe.

En Panamá y Santiago los entornos favorecedores de la elisión son el preconsonántico y el prepausal, al igual que en Barranquilla. El prepausal también favorece esta variante en San Juan. Vale la pena anotar los altos índices de elisión en el contexto prevocálico en San Juan, Panamá y Santiago, al igual que en Cuba, siendo este entorno el más favorecedor en los cubanos. Estos datos coinciden con los de Barranquilla, donde la elisión es norma junto con la aspiración.

Analicemos ahora el factor tonicidad de la sílaba, el cual también resulta importante en las distintas realizaciones de (s) en Barranquilla.

\begin{tabular}{|c|c|c|}
\hline & Tónica \% & Átona \% \\
\hline $\mathrm{s}$ & 17.1 & 14.7 \\
\hline $\mathrm{h}$ & 56.7 & 32.2 \\
\hline 0 & 25.7 & 52.9 \\
\hline $\mathrm{a}$ & 0.3 & 0.1 \\
\hline Número de casos & 1.378 & 1.822 \\
\hline
\end{tabular}

Tabla 8. Distribución de las variantes de (s) según tonicidad de la sílaba.

Nuevamente los resultados demuestran una distribución complementaria de la aspiración y la elisión; la primera se favorece en la sílaba tónica (56.7), mientras la segunda en la átona (52.9). La retención de (s) plena se favorece en la sílaba tónica, lo cual coincide con los resultados de Terrell (1979: 607) en la comunidad cubana: 19\% para la sílaba átona y 39 \% para la tónica, y los resultados de López Morales (1983: 46; 1992: 84) para San Juan de Puerto Rico: 27.7\% 
para la tónica y $13.3 \%$ para la átona. En esta comunidad también la sílaba tónica favorece la aspiración, al igual que en Barranquilla.

Finalmente, dentro de los factores lingüísticos, es importante analizar el valor morfológico de (s) para observar si en la comunidad de habla barranquillera este factor incide en la retención de (s) plena y en el debilitamiento. Se cuantificaron las (s) con valor morfológico de plural en las formas nominales: sustantivos y modificadores, artículos, adjetivos y demostrativos; y en las formas verbales, con valor de segunda persona de singular:

\begin{tabular}{|c|c|c|}
\hline & Nominal \% & Verbal \% \\
\hline $\mathrm{s}$ & 12.4 & 40.6 \\
\hline $\mathrm{h}$ & 37.5 & 43.7 \\
\hline 0 & 50 & 15.6 \\
\hline Número de casos & 1.239 & 32 \\
\hline
\end{tabular}

Tabla 9. Distribución de las variantes de (s) según el valor morfológico de (s)

En las formas nominales el valor morfológico de (s) no es un factor determinante en la retención; por el contrario, la frecuencia más alta de elisiones de (s) se registra en dichas formas con morfema de plural (50\%). En las formas verbales el morfema de persona sí parece cumplir una función en la retención de (s) en su forma plena [s] o aspirada [h]; los índices más bajos se registran en la elisión (15.6). Es probable que en las formas nominales la redundancia morfológica sea un factor que incida en los resultados, pues el hablante puede dejar una sola marca de plural: el morfema '-s', parte de éste en la forma 'es' > 'e' o la raíz de algunas formas, por ejemplo, el artículo plural 'los' (frente al singular 'el'), el indefinido 'algunos' (frente al singular 'algún'), elidiendo las demás: [lah |3wélta0] "las vueltas", [kosínah inteYrále0] "cocinas integrales", [lo0 5errúmbe0] "los derrumbes".

Además de los factores lingüísticos independientes, los sociales revelan resultados interesantes en la ciudad de Barranquilla. Analicemos las variables nivel educativo, sexo, edad y etnia.

\begin{tabular}{|c|c|c|c|}
\hline & Nivel 1, en \% & Nivel 2, en \% & Nivel 3, en \% \\
\hline $\mathrm{S}$ & 20.3 & 33.8 & 45.7 \\
\hline $\mathrm{h}$ & 30 & 37.5 & 32.3 \\
\hline 0 & 41 & 29.1 & 29.8 \\
\hline
\end{tabular}

Tabla 10. Distribución social de las variantes de (s) según el factor nivel educativo. 
Pese a que la retención de [s] plena no es norma en Barranquilla, la mayor frecuencia de esta variante se registra en el nivel educativo 3 (45.7), seguido del 2 (33.8), y la menor frecuencia se encuentra en el nivel 1. Es interesante ver cómo la aspiración es norma general en los tres niveles educativos, como reflejo de la regla que tipifica el español de Barranquilla, aunque el nivel 1 registra la menor frecuencia (30). En este nivel educativo la norma es la elisión. Los porcentajes más altos se hallan en este grupo (41\%), frente a un $29.1 \%$ en el nivel $2 \mathrm{y}$ un $29.1 \%$ en el 3. Nótese cómo estos dos últimos niveles comparten la misma frecuencia de uso de la elisión, mucho menor que el nivel 1. Veamos los otros factores.

\begin{tabular}{|c|c|c|}
\hline & Hombres, en $\%$ & Mujeres, en $\%$ \\
\hline $\mathrm{s}$ & 25.3 & 74.6 \\
\hline $\mathrm{h}$ & 47.9 & 52 \\
\hline 0 & 61 & 38 \\
\hline
\end{tabular}

Tabla 11. Distribución social de las variantes de (s) según el factor sexo.

Los resultados revelan un patrón sociolingüístico ya registrado en otras investigaciones tanto hispanas como no hispanas: la tendencia en las mujeres al uso de variantes estándares y no estigmatizadas, y no estándares y estigmatizadas en los hombres ${ }^{10}$. En Barranquilla

${ }^{10}$ Dicha diferenciación entre los sexos se ha interpretado a partir de la hipótesis según la cual las mujeres están más cercanas a las formas prestigiosas o son más sensibles a éstas. Esta hipótesis adopta la forma de tres principios del cambio lingüístico: 1) La conformidad lingüística de las mujeres, lo cual indica que para las variables lingüísticas estables, las mujeres muestran una tasa más baja de variantes estigmatizadas y una tasa más alta de variantes de prestigio que los hombres (Labov, 2001: 266); 2) en el cambio lingüístico desde arriba, las mujeres adoptan formas de prestigio en una tasa más alta que los hombres (Labov, 2001: 274); 3) en el cambio lingüístico desde abajo las mujeres usan con más alta frecuencia formas innovadoras que los hombres (Labov, 2001: 292). Trudgill (1974: 88-99) afirma que las mujeres están más influidas por las formas estándares y los hombres encabezan el uso de formas vernaculares; las razones de este hecho según el autor son las siguientes: 1) El tipo de apropiación: las variedades geográficas, étnicas y de clase social son el resultado de la distancia y las variedades de sexos lo son de la diferencia: se esperan diferentes atributos sociales y diferente comportamiento para los hombres y las mujeres; 2) la relación con redes sociales. Milroy (1987) demostró que el tipo de red social en la que participan los hablantes y la intensidad de su participación puede tener un efecto significativo en su comportamiento lingüístico. En Belfast la consecuencia de los patrones diferentes de empleo se traducía en que las redes de hombres eran considerablemente menos densas y múltiples; 3) las mujeres son más conscientes que los hombres de la significación social de las variables lingüísticas por varias razones: en primer lugar, por el papel que desempeñan en la crianza y educación de los niños; en segundo lugar, porque su posición social en 
las mujeres registran el mayor número de retenciones de [s] (74.6), y el menor número de elisiones (38) frente a una alta frecuencia de elisiones en los hombres (61) y un menor número de retenciones de [s] (25.3). En cuanto a la aspiración, las mujeres presentan mayor frecuencia que los hombres. Esta diferencia aspiración/elisión que marca la diferencia mujeres versus hombres, respectivamente, se encuentra en otras zonas del Caribe. En Panamá ocurre el mismo fenómeno: "Where women were shown to favor aspiration, male speakers appear to delete more often" (Cedergren, 1972: 63).

Analicemos ahora el factor edad.

\begin{tabular}{|c|c|c|c|c|}
\hline & Grupo 1, en \% & Grupo 2, en \% & Grupo 3, en \% & Grupo 4, en \% \\
\hline $\mathrm{s}$ & 16.3 & 21.3 & 32 & 30.3 \\
\hline $\mathrm{h}$ & 28.5 & 26 & 23.8 & 21.5 \\
\hline 0 & 25.1 & 26.2 & 25.1 & 23.5 \\
\hline
\end{tabular}

Tabla 12. Distribución social de las variantes de (s) según el factor edad.

El mayor número de retenciones de (s) plenas se encuentra en los grupos 3 y 4 . En la aspiración y la elisión se aprecian frecuencias análogas, lo cual corrobora el mantenimiento de estas dos reglas en la comunidad de habla barranquillera. Como se observó en la tabla 1 , ambas normas luchan su espacio en Barranquilla. La edad refleja la fortaleza de dichas reglas. No obstante, es importante señalar la mayor frecuencia de aspiraciones en el grupo 1, de jóvenes entre los 16 y los 20 años, seguido del grupo 2, personas entre los 30 y los 50 años. Si retomamos los datos de la retención de (s) plena se observan hechos interesantes: los grupos mayores, 3 y 4, de 51 a 65 años y de 66 años en adelante, respectivamente, se acercan en el uso de la forma estándar, en el mantenimiento de la (s), mientras los grupos jóvenes, 1 y 2 , se acercan en la regla de aspiración de (s).

la sociedad occidental ha sido tradicionalmente menos segura y por ello quizá han tenido que señalar su estatus social mediante el uso de la lengua, y en último lugar porque los hombres en este tipo de sociedad han sido valorados por lo que hacen (ocupación y ganancia de poder), mientras que las mujeres son valoradas por lo que dicen. A pesar de todos estos argumentos, la hipótesis del prestigio asociado a las mujeres no es totalmente firme, pues, como afirma Milroy (1987:202), quedan sin explicar algunas situaciones contrarias, por ejemplo, los casos de las esposas de hombres con ocupaciones de alto estatus que no imitan el modo de hablar de su esposo. La relación sexo-cambio lingüístico variará de comunidad en comunidad, quizá invirtiéndose los valores y los resultados. Ello dependerá del tipo de relaciones sociales creadas en el grupo, de las valoraciones y de la ideología. 
El factor etnia se revela interesante en la comunidad de habla barranquillera. Dentro de los informantes se registraron las entrevistas del grupo de palenqueros. Para este análisis seleccioné diez informantes, cinco de la etnia palenquera y cinco barranquilleros no palenqueros. Estos informantes poseen el mismo perfil en cuanto a las variables nivel educativo, edad y sexo. Esto con el fin de aislar la variable etnicidad y observar su significación en Barranquilla:

\begin{tabular}{|c|c|c|c|}
\hline & Palenqueros, en \% & Barranquilleros, en \% & Número de casos \\
\hline S & 23.9 & 76 & 142 \\
\hline H & 52.8 & 47.1 & 352 \\
\hline 0 & 54.8 & 45.1 & 501 \\
\hline A & 100 & 0 & 5 \\
\hline
\end{tabular}

Tabla 13. Distribución social de las variantes de (s) según el factor etnia.

Los resultados son significativos. La retención de (s) plena es mayor en los barranquilleros, mientras la elisión es mayor en los palenqueros. La regla de aspiración registra un aumento en la etnia palenquera, lo cual puede reflejar un fenómeno de hipercorrección en este grupo, si tenemos en cuenta que la aspiración es la norma de prestigio en Barranquilla con respecto a la elisión. Los inmigrantes palenqueros tenderían a llevar más allá la norma del habla barranquillera como una muestra de identificación con la comunidad receptora. Esta acomodación se reitera si analizamos los resultados de la asimilación total de (s) al segmento siguiente, la cual es norma en el habla de los habitantes de San Basilio de Palenque. Pese a que el uso es categórico en la etnia palenquera, frente a los barranquilleros, el número de asimilaciones es mínimo, cinco casos solamente, frente a 34 casos de [s], 186 de [h]. Esto demuestra un cambio lingüístico en marcha en el grupo palenquero que refleja un proceso de desdialectalización motivado por las constricciones sociales. Esto se corrobora si vemos el caso de la variable (-r) implosiva, la cual no es objeto de análisis aquí por causa de espacio, pero que revela el mismo patrón en cuanto a su variante lateralizada, la cual sólo se registró en los hablantes mayores de 60 años y con una frecuencia muy baja (tres a cuatro casos). Tanto la asimilación plena de (s) como la lateralización de (l) son variantes estigmatizadas en la ciudad de Barranquilla y esta comunidad identifica estas variantes como pertenecientes al habla palenquera, por lo cual es evaluada de modo estigmatizado, como lo revelan las pruebas de actitudes y creencias (Rodríguez Cadena, 2004: 118). 
Pese a la cohesión étnica y cultural de la comunidad palenquera, este grupo demuestra un sentido de pertenencia a la ciudad de Barranquilla, como se anotó al inicio de este ensayo, pues comparten la variedad dialectal del Caribe hispánico y forman parte de la identidad caribeña. Veamos ahora la distribución de variantes según origen.

\begin{tabular}{|c|c|}
\hline Variante & $\%$ \\
\hline $\mathrm{s}$ & 43.9 \\
\hline $\mathrm{h}$ & 39.1 \\
\hline 0 & 16.8 \\
\hline
\end{tabular}

Tabla 14. Comunidad sanandresana. Variable (s).

Como se observa, en la comunidad sanandresana la variable (s) plena tiene una alta frecuencia, al lado de la aspiración. Esto refleja una diferencia con los nativos en Barranquilla (ver tabla 1), en quienes la aspiración y la elisión son la norma.

\section{Variable (r)}

\begin{tabular}{|c|c|}
\hline Variantes & $\begin{array}{c}\text { Más de } 12 \text { años de residencia en } \\
\text { Barranquilla, en } \%\end{array}$ \\
\hline $\mathrm{r}$ & 78 \\
\hline $\mathrm{a}$ & 5.2 \\
\hline 0 & 15.8 \\
\hline
\end{tabular}

Tabla 15. Comunidad cordobesa. Variable (r). Estilo semiformal.

Aquí se observa que los inmigrantes que tienen más de doce años de residir en Barranquilla pronuncian la / $\mathrm{r}$ / en su variante plena [r] en un alto índice, (78\%), mientras que la asimilación plena al segmento siguiente, norma del habla cordobesa, posee un porcentaje mínimo, (5.2\%). Esto indica un desplazamiento del dialecto cordobés y una acomodación a las normas lingüísticas de la comunidad de habla barranquillera.

\begin{tabular}{|c|c|c|}
\hline Variantes & $\begin{array}{c}\text { Más de 12 años } \\
\text { de residencia en } \\
\text { Barranquilla, en \% }\end{array}$ & $\begin{array}{c}\text { Menos de un año de } \\
\text { residencia en Barranquilla, } \\
\text { en \% }\end{array}$ \\
\hline $\mathrm{r}$ & 57.2 & 42.7 \\
\hline $\mathrm{a}$ & 37.5 & 62.5 \\
\hline 0 & 35.2 & 64.7 \\
\hline
\end{tabular}

Tabla 16. Comunidad bolivarense. Variable (r). Estilo semiformal. 
Al igual que en la comunidad cordobesa, en la de Bolívar, inmigrante en la ciudad de Barranquilla, ocurre un proceso de desdialectalización. Como se observa en la tabla 16, los inmigrantes que tienen más de doce años de residir en Barranquilla pronuncian la /r/ en su variante plena [r] más frecuentemente que la variante asimilada [a]; se aprecia un $57.2 \%$ para la primera frente a un $37.5 \%$ para la segunda. Mientras que en los inmigrantes que tienen menos de un año en la ciudad mantienen un porcentaje alto de asimilaciones: $62.5 \%$, frente a menos variantes plenas, $62.5 \%$.

\begin{tabular}{|c|c|}
\hline Variantes & $\begin{array}{c}\text { Más de } 12 \text { años de residencia en } \\
\text { Barranquilla, en \% }\end{array}$ \\
\hline $\mathrm{r}$ & 95.3 \\
\hline $\mathrm{a}$ & 1.5 \\
\hline 0 & 3 \\
\hline
\end{tabular}

Tabla 17. Comunidad sucreña. Variable (r). Estilo semiformal.

En la comunidad sucreña se repite el mismo patrón. Los inmigrantes que tienen más de doce años de residencia han casi eliminado la norma dialectal de Sucre de la asimilación total de /r/; el porcentaje es mínimo, 1.5\%, frente a un alto índice, 95.3\%, de /r/ plenas.

Los procesos de desplazamiento y mantenimiento de dialectos se corroboran en el análisis sociolingüístico. En la situación de contacto dialectal se ven implicados otros contactos de tipo social y cultural; ocurren encuentros lingüísticos, pero también encuentros de culturas. A estos procesos se suman los factores psicosociales que inciden de modo determinante en los cambios que ocurren en el habla de las comunidades. Ciertamente las actitudes y las creencias se constituyen en impulsadores de los procesos de acomodación lingüística a los que se ven abocados los inmigrantes. La continuidad de este estudio, especialmente en la profundización de esta complejidad, es una tarea inaplazable.

\section{Bibliografía}

Alba, Orlando. (1990). Variación fonética y diversidad social en el español dominicano de Santiago. Santiago: Pontificia Universidad Católica Madre y Maestra.

Arteta, María Eulalia \& Atencio, Ramón. (1990). Perfil socio-económico de Barranquilla. Barranquilla: CERES-Universidad del Norte.

Becerra, Servio. (1985). Fonología de las consonantes implosivas en el español urbano de Cartagena de Indias (Colombia). Bogotá: Instituto Caro y Cuervo. 
Cedergren, Herietta. (1973). The Interplay of Social and Linguistic Factors in Panama. Cornell University. Tesis doctoral inédita.

DANE (1993). Censo del Departamento del Atlántico. Barranquilla.

Dorta, Josefa. (1997). "Datos acústicos y percepción de la 0 adherente de Canarias y de la pre-palatal castellana", en Almeida \& Dorta. (eds.). Contribuciones al estudio de la lingüística hispánica. España: Montesinos, 57-71.

Friedemann, Nina. (1992). “Huellas de africanía en Colombia”, en Thesaurus, 47,$3 ; 543-559$.

Labov, William. (2001). Principles of Linguistic Change. Vol 2: Social factors. Massachusetts: Blackwell.

Lafford, Bárbara. (1988). "La sincronía dinámica en el español de Cartagena", en E. Luna Traill (ed.), Actas del VI Congreso Internacional de la Asociación de lingüística y Filología de la América Latina. México: Universidad Autónoma de México; 509-526.

López Morales, Humberto. (1983). Estratificación social del español de San Juan de Puerto Rico. México: Universidad Autónoma de México. .(1992). El español del Caribe. Madrid: MAPFRE.

Manco Bermúdez, Dino \& Watnik Barón, José. (1996). Miembros sobresalientes: comunidad judía en Barranquilla. Barranquilla: Man Comunicaciones.

Martín Butragueño, Pedro. (2002). “Contacto de dialectos y cambio lingüístico", ms.

Milroy, Lesley. (1987). Language and Social Networks. Oxford: Basil Blackwell.

.(1982). "Social network and linguistic focusing", en S. Romaine (ed.), Sociolinguistic Variation in Speech Communities. London: Edward Arnold, pp. 141-152.

Montes Giraldo, José Joaquín. (1987). Dialectología general e hispanoamericana. Bogotá: Instituto Caro y Cuervo. .(2000). Otros estudios sobre el español de Colombia. Bogotá: Instituto Caro y Cuervo.

Navarro, Tomás. (1974). El español de Puerto Rico. Río Piedras: Universidad de Puerto Rico.

Posada Carbó, Eduardo. (1987). Una invitación a la historia de Barranquilla. Bogotá: CEREC. (1998). El Caribe colombiano. Bogotá: Banco de la República.

Rodríguez Cadena, Yolanda. (2004). "Variación y cambio lingüístico en el Caribe colombiano: estudio sobre la ciudad de Barranquilla", en Historia y Cultura, 1, pp. 103-120.

Terrell, Tracy. (1979). "Final /s/ in cuban spanish", en Hispania, 62, 4, pp. 599-612.

Trudgill, Peter. (1974). Sociolinguistics: an introduction to language and society. London: Penguin Books. 
